$\xi_{p}=1$

\title{
Analysis and Modeling of Generic Mac Protocols in Wireless Sensor Network
}

\author{
Praneeta R Sheelavantar ${ }^{1}$, Shreedhar A Joshi' ${ }^{2}$, Gopal A Bidkar ${ }^{3}$ \\ ${ }^{1,3}$ Department of Electronics and Communication ${ }^{1,3}$ SDM College of Engineering \& Technology Dharwad 580002. Karnataka, India. \\ ${ }^{2}$ Department of Electronics and Communication, ${ }^{2}$ SDM College of Engineering \& Technology Dharwad 580002. Karnataka, India. \\ *Corresponding author E-mail: ${ }^{l}$ pranusheelavantar94@gmail.com, ${ }^{2}$ shreedhar.joshi912@gmail.com
}

\begin{abstract}
Nowadays Wireless Sensor Networks are used everywhere, which plays an important role in recording the physical conditions of the environment and organizing the data collected at a central location. WSN has many applications in fields such as military, home automation, industries, commercial, battle field, food safety etc. This paper presents the analysis of Generic MAC Protocols with different routing protocols using Qualnet 7.4 simulator. The simulation result shows that comparing with other MAC protocols SLOT CSMA/CA gives better performance. Total unicast message sent, received, average end-to-end delay, average jitter, average throughput, energy consumed, packets received and lost are analysed for each routing protocol.
\end{abstract}

Keywords: CSMA, CSMA/CA, SLOT CSMA, SLOT CSMA/CA, QualNet.

\section{Introduction}

Wireless Sensor Networks (WSNs) have a wide variety of applications ranging from body area health monitoring to wide-range environmental surveillance. WSN is having responsibility of measuring environmental variables using sensors for pre-processing and transmission of the gained data to the sink i.e agent is engaged in collecting the information developed from sensor nodes. WSNs have few resources like memory, computation capability and available energy [1]. From Bluetooth to cell phone all works on a network. Networks have different size, shape and complexity [2]. In designing the network protocols many efforts are made in order to fulfil the essential needs of the WSNs, mainly about the Medium Access Control (MAC) layer [1]. The purpose of a MAC layer is providing mechanisms to grant certain wireless nodes for sharing the wireless channel medium and access the network. The major responsibility of the MAC layer is to avoid collisions among nodes and providing fair medium allocation between the nodes. MAC layer shows an essential role in the energy efficiency of communication [1].

Functions of MAC Layer [11]:

- The assembly of data into a frame for transmission by attaching a header field comprises of addressing information and a trailer field for error detection.

- The disassembly of a received frame for the selection of addressing and error control information to perform address recognition and error detection and recovery.
- The regulation of access to the shared transmission medium in a way compatible with the performance requirements of the supported application.

\section{Literature Survey}

A generic framework is proposed to model a vast area of MAC protocols by using AMCs and evaluated energy consumption, latency, reliability and showed that model gives exact estimation for the context of low throughput applications [1]. The energy efficiency of the IEEE 802.15.4 MAC layer in dense networks is calculated and it can be used for communication in dense data gathering networks [3]. Non-persistent CSMA with back-off is modelled using IEEE 802.15.4 MAC layer, throughput and energy consumption of the contention access period are evaluated and also energy efficiency is improved by closing down the radio between transmissions [4]. Markov Chains are used for modelling IEEE 802.15.4 MAC protocol, calculated throughput and energy consumption [5]. In [6], Markov Chains are used for modelling IEEE 802.15.4 MAC layer and evaluated reliability, delay and energy consumption and exposed that MAC parameters and collision probability are two main execution metrics that are mainly needed. For scaling down unessential data transmission, a spatial correlation between sensor nodes on MAC layer is proposed [7]. In [8], duty-cycle, energy efficiency, and latency are analysed for MAC protocols having lesser data-rate WSNs with respect to network parameters like transceiver and network density. 


\section{Scenario Description}

The scenario shown below is created and analysed in QualNet 7.4 simulator. "QualNet is a state-of-the-art simulator for large, heterogeneous networks and the distributed applications that execute on networks" [9].

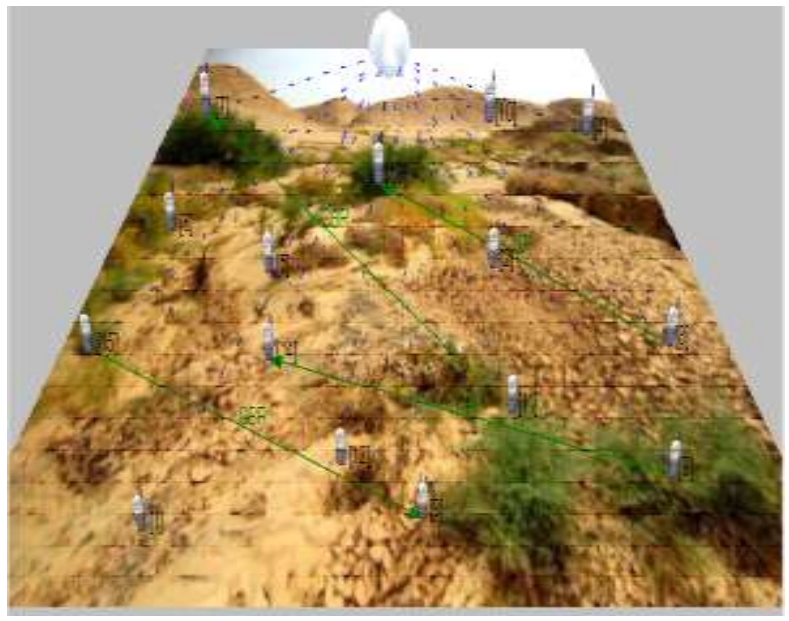

Figure 1: Scenario created in QualNet X-Y view

The simulation model consists of 15 nodes which are connected to one wireless subnet having terrain dimension $700 \mathrm{~m} \times 700 \mathrm{~m}$, in which area is considered as flat and range of attitude above sea level is $1500 \mathrm{~m}$. IEEE standard 802.15.4 radio type MAC protocol is used for simulation. The energy model used is Mica-Motes and communication channel having $2.4 \mathrm{GHz}$ frequency using O-QPSK modulation scheme. Constant Bit Rate (CBR) type of traffic is used. The source and destination for every CBR is selected in a random manner. Simulated execution time for flow of data for every source and destination is constant to 300 seconds for whole simulation. Mobility interval for all CBR is $1 \mathrm{sec}$. Figure 1 displays the scenario created in QualNet X-Y view.

Figure 2 shows the scenario created in QualNet 3D view.

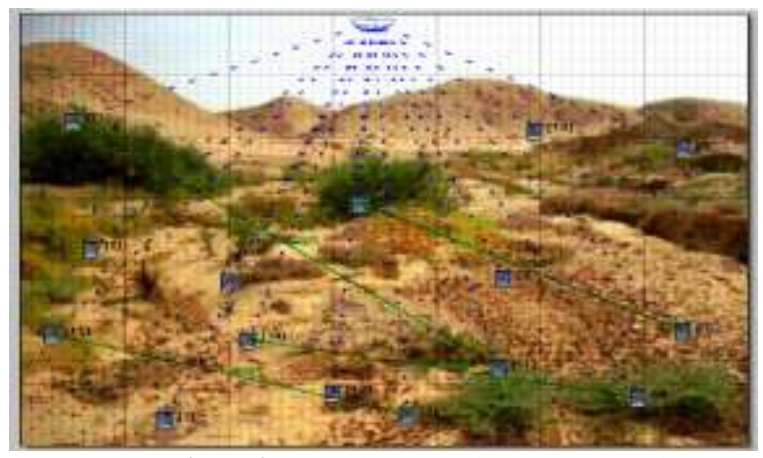

Figure 2: Scenario created in QualNet 3D view

\section{Simulation Environment}

The performance of Generic MAC protocols such as CSMA, SLOT CSMA, CSMA/CA and SLOT CSMA/CA are evaluated using AODV, Bellman-Ford and DSR routing protocols. This is simulated by conducting Batch Experiments in a Non-Interactive Run mode. Table I shows the summarization of parameters used in simulation setup. In this data is collected for eleven performance metrics namely: total unicast message sent, total unicast message received, throughput, end to end delay, average jitter, packets from networks, packets lost and energy consumption. Figure 3 shows the simulation of scenario.

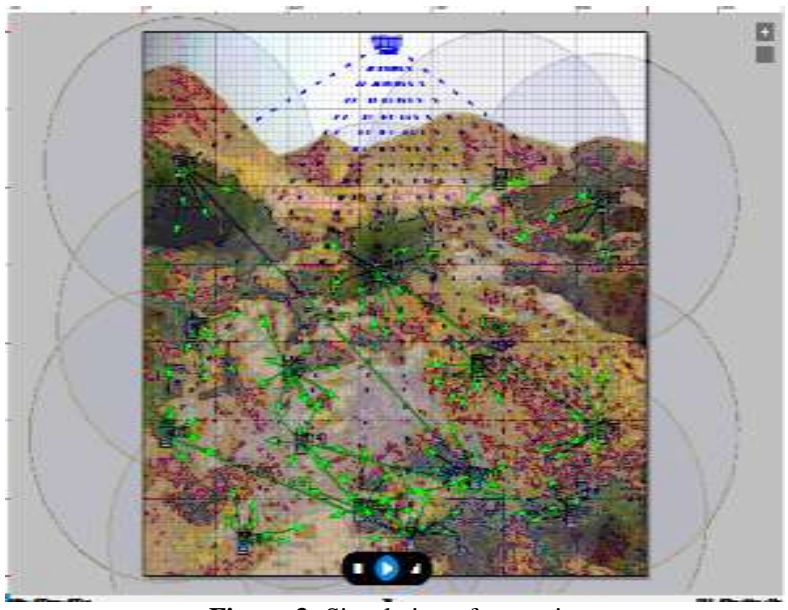

Figure 3: Simulation of scenario

Table I: Parameters Used in Simulation

\begin{tabular}{|l|l|}
\hline Parameters & Value \\
\hline Terrain Dimensions & $700 \times 700 \mathrm{~m}$ \\
\hline Altitude above sea level & $1500 \mathrm{~m}$ \\
\hline Simulation time & $300 \mathrm{sec}$ \\
\hline No. of Nodes & 15 \\
\hline Mobility Interval & $10 \mathrm{sec}$ \\
\hline No. of Channel & 1 \\
\hline No. of CBRs & 4 \\
\hline MAC Protocol & Generic MAC \\
\hline Channel Access Mode & CSMA, SLOT CSMA, \\
\hline CSMA/CA, SLOT CSMA/CA \\
\hline Node Placement & Random \\
\hline Network Protocol & CBR \\
\hline Routing Protocol & IPv4 \\
\hline Energy Model & AODV, BELLMAN-FORD, DSR \\
\hline Temperature & Mica-Motes \\
\hline Antenna Model & $290 \mathrm{~K}$ \\
\hline Path loss model & Omni directional \\
\hline
\end{tabular}

\section{Results and Analysis}

The results of all Generic MAC Protocols are analysed and compared by using three routing protocols.

\subsection{CSMA}

The channel is checked by a node before sending a packet to the destination. If the channel is found idle, packets are sent immediately to the destination or else the node starts back-off timer. When the back-off timer elapses, again the channel is sensed by the node. If the channel is idle, packets are sent immediately; or else the back-off timer is rescheduled by the node. The same process is carried out until successful transmission of the packets or until it attains largest number of retransmission. The back-off timer is selected randomly from a time interval which is exponentially incremented with each trial of retransmission. The packets are dropped if the maximum number of retransmission is reached [10]. Table II shows the comparison of CSMA protocol using three different routing protocols. 
Table II: Comparison of CSMA Protocol

\begin{tabular}{|l|l|l|l|}
\hline & AODV & BELLMAN-FORD & DSR \\
\hline $\begin{array}{l}\text { Total Unicast } \\
\text { message sent } \\
\text { (msgs) }\end{array}$ & 299 & 299 & 299 \\
\hline $\begin{array}{l}\text { Total Unicast } \\
\text { message } \\
\text { received(msgs) }\end{array}$ & 298 & 262 & 299 \\
\hline Delay (sec) & 0.153 & 0.177501 & 0.1338 \\
\hline $\begin{array}{l}\text { Throughput } \\
\text { (bits/sec) }\end{array}$ & 4084.74 & 3603 & 4100.88 \\
\hline Jitter (sec) & 0.02930 & 0.035336 & 0.06887 \\
\hline $\begin{array}{l}\text { Transmit mode } \\
\text { (mWh) }\end{array}$ & 0.0658 & 0.00723 & 0.00514 \\
\hline $\begin{array}{l}\text { Receive mode } \\
\text { (mWh) }\end{array}$ & 0.29735 & 0.961653 & 0.44178 \\
\hline Idle mode (mWh) & 1.06661 & 0.746005 & 1.01767 \\
\hline $\begin{array}{l}\text { Sleep mode } \\
\text { (mWh) }\end{array}$ & 0 & 0 & 0 \\
\hline $\begin{array}{l}\text { Packets from } \\
\text { network }\end{array}$ & 321 & 37 & 9 \\
\hline Packets lost & 1 & 28 & 2 \\
\hline
\end{tabular}

In this protocol, DSR receives all unicast message sent by the client, delay is less, and throughput is more compared to other two whereas jitter is less in AODV. Energy consumed is less in DSR compared to AODV and Bellman-Ford. More number of packets is received from the upper layer in AODV and packet loss is less.

\subsection{SLOT CSMA}

In SLOT CSMA, the time line is divided into equal slots. This protocol is similar to CSMA except that only at the beginning of a slot a node attempts to transmit. When new time slot begins, channel sensing is done. The node sends the packet on the channel if it is found to be idle else packets are rescheduled to some randomly chosen time in the future using the back-off timer [10]. Table III shows the comparison of SLOT CSMA Protocol using three different routing protocols.

Table III: Comparison of SLOT CSMA Protocol

\begin{tabular}{|l|l|l|l|}
\hline & AODV & $\begin{array}{l}\text { BELLMAN- } \\
\text { FORD }\end{array}$ & DSR \\
\hline $\begin{array}{l}\text { Total Unicast message sent } \\
\text { (msgs) }\end{array}$ & 299 & 299 & 299 \\
\hline $\begin{array}{l}\text { Total Unicast message } \\
\text { received(msgs) }\end{array}$ & 41 & 24 & 7 \\
\hline Delay (sec) & 5.83275 & 100.218 & 52.5725 \\
\hline Throughput (bits/sec) & 643.441 & 356.825 & 107.986 \\
\hline Jitter (sec) & 1.69375 & 6.44696 & 14.0267 \\
\hline Transmit mode (mWh) & 0.11719 & 0.034118 & 0.35746 \\
\hline Receive mode (mWh) & 0.15186 & 0.215641 & 0.23285 \\
\hline Idle mode (mWh) & 1.1201 & 1.11229 & 0.97376 \\
\hline Sleep mode (mWh) & 0 & 0 & 0 \\
\hline Packets from network & 570 & 40 & 395 \\
\hline Packets lost & 40 & 0 & 201 \\
\hline
\end{tabular}

In this protocol, more unicast messages are received from the client, delay and jitter is less, throughput is more in AODV and packet loss is less in Bellman-Ford.

\subsection{CSMA/CA}

In CSMA/CA, before transmitting the packets the channel is checked by the node. The back-off timer is lapsed if the channel is busy. If it is found idle, the back-of timer is restarted. The back-off timer will work in collision-avoidance period and packets are carried on the channel during other periods [10]. Table IV shows the comparison of CSMA/CA protocol using three different routing protocols.

Table IV: Comparison of CSMA/CA Protocol

\begin{tabular}{|l|l|l|l|}
\hline & AODV & $\begin{array}{l}\text { BELLMAN- } \\
\text { FORD }\end{array}$ & DSR \\
\hline $\begin{array}{l}\text { Total Unicast message sent } \\
\text { (msgs) }\end{array}$ & 299 & 299 & 299 \\
\hline $\begin{array}{l}\text { Total Unicast message } \\
\text { received(msgs) }\end{array}$ & 299 & 236 & 68 \\
\hline Delay (sec) & 0.1354 & 0.109051 & 11.5398 \\
\hline Throughput (bits/sec) & 4100.04 & 3233.88 & 939.485 \\
\hline Jitter (sec) & 0.07417 & 0.091960 & 1.92347 \\
\hline Transmit mode (mWh) & 0.00594 & 0.009739 & 0.06369 \\
\hline Receive mode (mWh) & 0.42236 & 1.28899 & 0.42261 \\
\hline Idle mode (mWh) & 1.02744 & 0.574427 & 1.00228 \\
\hline Sleep mode (mWh) & 0 & 0 & 0 \\
\hline Packets from network & 25 & 44 & 293 \\
\hline Packets lost & 0 & 243 & 149 \\
\hline
\end{tabular}

In this protocol, comparing three routing protocols AODV shows better performance in terms of messages received from the client, throughput, jitter, energy consumption and packet lost. Delay is less in Bellman-Ford.

\subsection{SLOT CSMA/CA}

This protocol is same as CSMA/CA but depending on the back-off timer time line slots are predetermined. When node wants to transmit packets, before transmitting, the channel is sensed randomly using back-off timer collision avoidance period for checking out a clear channel [10]. Table V shows the comparison of SLOT CSMA/CA protocol using three different routing protocols.

In this protocol, both Bellman-Ford and DSR receives all messages sent by the client, delay is less in AODV, throughput and jitter are better in DSR, less energy is consumed in Bellman-Ford. No packets are lost in any routing protocols.

Table V: Comparison of SLOT CSMA/CA Protocol

\begin{tabular}{|l|l|l|l|}
\hline & AODV & $\begin{array}{l}\text { BELLMAN- } \\
\text { FORD }\end{array}$ & DSR \\
\hline $\begin{array}{l}\text { Total Unicast message } \\
\text { sent (msgs) }\end{array}$ & 299 & 299 & 299 \\
\hline $\begin{array}{l}\text { Total Unicast message } \\
\text { received(msgs) }\end{array}$ & 289 & 299 & 299 \\
\hline Delay (sec) & 0.0992 & 0.1072 & 0.4799 \\
\hline Throughput (bits/sec) & 4097.39 & 4098.58 & 4242.84 \\
\hline Jitter (sec) & 0.35 & 0.0176 & 0.0385 \\
\hline Transmit mode (mWh) & 0.03565 & 0.005669 & 0.0393 \\
\hline Receive mode (mWh) & 0.04920 & 0.012115 & 0.0506 \\
\hline Idle mode (mWh) & 1.20892 & 1.24123 & 1.2065 \\
\hline Sleep mode (mWh) & 0 & 0 & 0 \\
\hline Packets from network & 7 & 41 & 45 \\
\hline Packets lost & 0 & \multicolumn{2}{|l}{0} \\
\hline
\end{tabular}

\section{Conclusion}

The scenario is created using QualNet 7.4 simulator using IEEE 802.15.4 standard. Different quality of services like delay, jitter, throughput, energy consumption, unicast messages sent and 
received, packets from network and packets lost have been analysed using different routing protocols for different Generic MAC protocols using Mica-Motes energy model. Comparing all MAC protocols, SLOT CSMA/CA is more efficient where no packets are lost.

\section{Acknowledgement}

Our sincere thanks to the Principal, HOD, PG co-ordinator, guide and other teaching and non-teaching staff members of E\&C department, SDMCET, Dharwad who have helped to carry-out this work.

\section{References}

[1] Fayçal Ait Aoudia, Matthieu Gautier, Michele Magno, Senior Member, IEEE, Olivier Berder, and Luca Benini, Fellow, IEEE, ACM, "A Generic Framework for Modeling MAC Protocols in Wireless Sensor Networks", IEEE/ACM TRANSACTIONS ON NETWORKING, VOL. 25, NO. 3, JUNE 2017.

[2] Nimarta Sidhu, Paramjeet Singh and Shaveta Rani, "Improved Optimal Slotted CSMA/CA Protocol", International Journal of Computer Applications (0975 - 8887) Volume 79 - No6, October 2013.

[3] B. Bougard, F. Catthoor, D. C. Daly, A. Chandrakasan, and W. Dehaene, "Energy efficiency of the IEEE 802.15.4 standard in dense wireless microsensor networks: Modeling and improvement perspectives," in Proc. Conf. Design, Autom. Test Eur. (DATE), vol. 1. Mar. 2005, pp. 196-201.

[4] I. Ramachandran, A. K. Das, and S. Roy, "Analysis of the contention access period of IEEE 802.15.4 MAC," ACM Trans. Sensor Netw., vol. 3, no. 1, Mar. 2007, Art. no. 4.

[5] S. Pollin et al., "Performance analysis of slotted carrier sense IEEE 802.15.4 medium access layer," IEEE Trans. Wireless Commun., vol. 7, no. 9, pp. 3359-3371, Sep. 2008.

[6] P. Park, P. D. Marco, P. Soldati, C. Fischione, and K. H. Johansson, "A generalized Markov chain model for effective analysis of slotted IEEE 802.15.4," in Proc. IEEE Int. Conf. Mobile Adhoc Sensor Syst., Oct. 2009, pp. 130-139.

[7] M. C. Vuran and I. F. Akyildiz, "Spatial correlation-based collaborative medium access control in wireless sensor networks," IEEE/ACM Trans. Netw., vol. 14, no. 2, pp. 316-329, Apr. 2006.

[8] K. Langendoen and A. Meier, "Analyzing MAC protocols for low data-rate applications," ACM Trans. Sensor Netw., vol. 7, no. 1, pp. $1-34,2010$.

[9] Sorout Dinesh and Goyal Sonal, "Qualnet Simulator", International Journal of Information \& Computation Technology. ISSN 0974-2239 Volume 4, Number 13 (2014), pp. 1349-1354,

[10] QualNet 6.1Wireless Model Library, Scalable Network Technologies, Inc. September 2012.

[11] Kazem Sohraby, Daniel Minoli, and Taieb Znati, "Wireless Sensor Networks: Technology, Protocols, and Applications" Copyright (C) 2007 John Wiley \& Sons, Inc. 